\title{
Phase diagram of the three dimensional Thirring model - A Monte Carlo study
}

\author{
Stavros Christofi \\ Frederick Institute of Technology, CY-1303 Nicosia, Cyprus \\ eng.csdfit.ac.cy
}

\section{Simon Hands}

Physics Department, Swansea University, Singleton Park, Swansea SA2 8PP, U.K.

s.hands@swan.ac.uk

\section{Costas Strouthos*}

Harvard-MIT (HST) Martinos Center for Biomedical Imaging, Massachusetts General Hospital,

Harvard Medical School, Charlestown, MA 02129, U.S.A.

cstrouthedeas.harvard.edu

Certain approximate solutions of the continuum Schwinger-Dyson Equations (SDEs) predict chiral symmetry breaking in the $3 \mathrm{~d}$ Thirring model when the number of fermion flavors $N_{f}<4.32$ [1] whereas others predict symmetry breaking for all $N_{f}$ [2]]. Our results from Monte Carlo simulations with $N_{f}=6$, predict a second order chiral phase transition. The critical coupling in this case corresponds to an ultra-violet fixed point of the renormalization group defining a non-trivial continuum limit. Further, our numerical simulations provide an estimate for the critical number of fermion flavors, $N_{f c} \approx 6.5$.

XXIVth International Symposium on Lattice Field Theory

July 23-28, 2006

Tucson, Arizona, USA

\footnotetext{
* Speaker.
} 


\section{Introduction}

The study of quantum field theories in which the ground state shows a sensitivity to the number of fermion flavors $N_{f}$ is intrinsically interesting. According to certain approximate solutions of continuum SDEs the $3 \mathrm{~d}$ Thirring model as well as $\mathrm{QED}_{3}$ display this phenomenon. The Thirring model is a theory of fermions interacting via a current contact interaction:

$$
\mathscr{L}=\bar{\psi}_{i}(\not \partial+m) \psi_{i}+\frac{g^{2}}{2 N_{f}}\left(\bar{\psi}_{i} \gamma_{\mu} \psi_{i}\right)^{2},
$$

where $\psi_{i}, \bar{\psi}_{i}$ are four-component spinors, $m$ is a parity-conserving bare mass, and the index $i$ runs over $N_{f}$ distinct fermion flavors. In the chiral limit the Lagrangian of the model has a continuous $U(1)$ chiral symmetry. Since the coupling $g^{2}$ has mass dimension -1 , naive power-counting suggests that the model is non-renormalisable. However, as has been suspected for many years [3, 4. 5], an expansion in powers of $1 / N_{f}$, rather than $g^{2}$, is exactly renormalisable and the model has a well-defined continuum limit corresponding to a UV-stable fixed point of the renormalization group. After the introduction of an auxiliary vector field, the above Lagrangian can be rewritten as:

$$
\mathscr{L}_{\text {aux }}=\bar{\psi}_{i}(\not \partial+i \not A+m) \psi_{i}+\frac{N_{f}}{2 g^{2}}\left(A_{\mu}\right)^{2} .
$$

The $1 / N_{f}$ expansion may not, however, describe the true behavior of the model, particularly for small $N_{f}$. Chiral symmetry breaking is forbidden at all orders in $1 / N_{f}$, and yet may be predicted by a self-consistent approach such as SDEs [1] 2]. SDEs solved exactly in the limit $g^{2} \rightarrow \infty$ [1] show that chiral symmetry is broken for $N_{f}<N_{f c} \simeq 4.32$. This is close to certain predictions of $N_{f c}$ for the non-trivial IR behavior in $\mathrm{QED}_{3}$ [6]. Based on these results, at $N_{f c}$ the model is expected to undergo an infinite order or conformal phase transition, originally discussed by Miranskii et al in the context of quenched QED $_{4}$ [䜣]. Using a different sequence of truncations Hong and Park [2] found chiral symmetry breaking for all $N_{f}$. Previous Monte Carlo simulations [8, 9, 10, 11] provided evidence that for $N_{f} \leq 5$ the model is in the chirally broken phase at strong enough coupling. Here, we present preliminary results based on numerical simulations with $N_{f}=2, \ldots, 18$ in an effort to estimate $N_{f c}$.

\section{Lattice Model}

The lattice action we have used is based on [8] and is as follows:

$$
\begin{aligned}
S= & \frac{1}{2} \sum_{x \mu i} \bar{\chi}_{i}(x) \eta_{\mu}(x)\left(1+i A_{\mu}(x)\right) \chi_{i}(x+\hat{\mu})+\text { h.c. } \\
& +m \sum_{x i} \bar{\chi}_{i}(x) \chi_{i}(x)+\frac{N}{4 g^{2}} \sum_{x \mu} A_{\mu}(x)^{2} \\
\equiv & \sum_{x y i} \bar{\chi}_{i}(x) M[A, m](x, y) \chi_{i}(y)+\frac{N}{4 g^{2}} \sum_{x \mu} A_{\mu}(x)^{2}
\end{aligned}
$$

where $\chi, \bar{\chi}$ are staggered fermion fields and the flavor index $i$ runs from 1 to $N$. We have introduced $M[A, m]$ for the fermionic bilinear, which depends on both the auxiliary field defined on the 

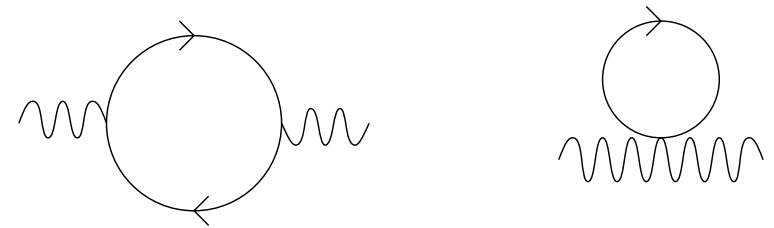

Figure 1: Diagrams contributing to vacuum polarization in lattice QED.

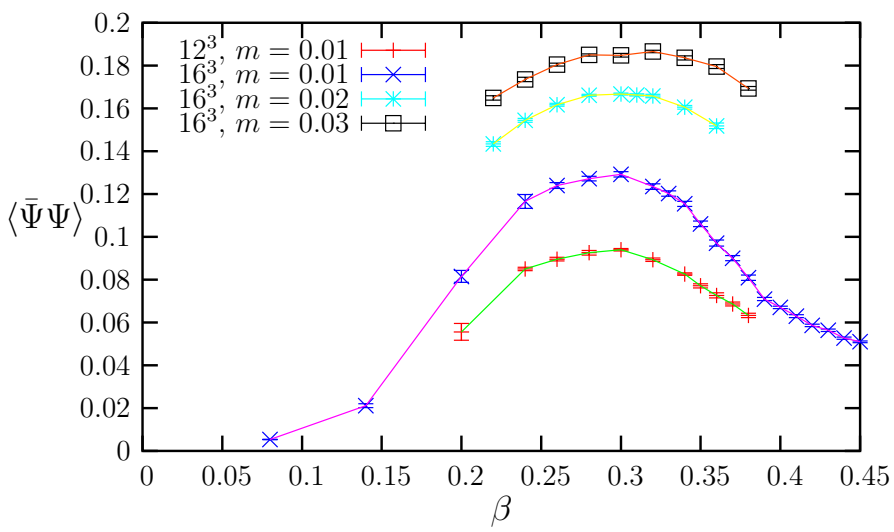

Figure 2: Chiral condensate vs. $\beta$ for $N_{f}=6$ near $\beta_{\text {lim }}$.

lattice links and the bare mass $m$. In three dimensions $N$ staggered fermion describe $N_{f}=2 N$ four-component continuum species. We employed the Hybrid Monte Carlo (HMC) algorithm to simulate even $N_{f}$ and we used the Hybrid Molecular Dynamics (HMD) algorithm for odd or noninteger $N_{f}$. In the HMD simulations we used small enough fictitious time step $\Delta \tau \leq 0.0025$ and ensured that the $O\left(N^{2} \Delta \tau^{2}\right)$ systematic errors in the molecular dynamics steps were smaller than the statistical errors of the various observables.

\section{Critical Number of Fermion Flavors}

In this section, we summarize the discussion presented in [9] regarding the non-conservation of the vector current at strong coupling in the lattice Thirring model of eq. 2.1. At leading order in $1 / N$ the vector propagator receives an extra non-transverse contribution from vacuum polarization, essentially due to the absence of the second diagram in Fig. 1. It should be noted that in noncompact $\mathrm{QED}_{3}$ both diagrams are present and a cancellation of $\mathrm{O}\left(a^{-1}\right)$ contributions from the two diagrams in Fig. 1 1 occurs. In the 3d Thirring model however, the effect of the absence of the second diagram can be absorbed into a redefinition of the coupling:

$$
g_{R}^{2}=\frac{g^{2}}{1-g^{2} J(m)},
$$

where $J(m)$ is the value of the integral contributed from the second diagram in the above figure. The physics described by continuum $1 / N_{f}$ perturbation theory occurs for the range of couplings 


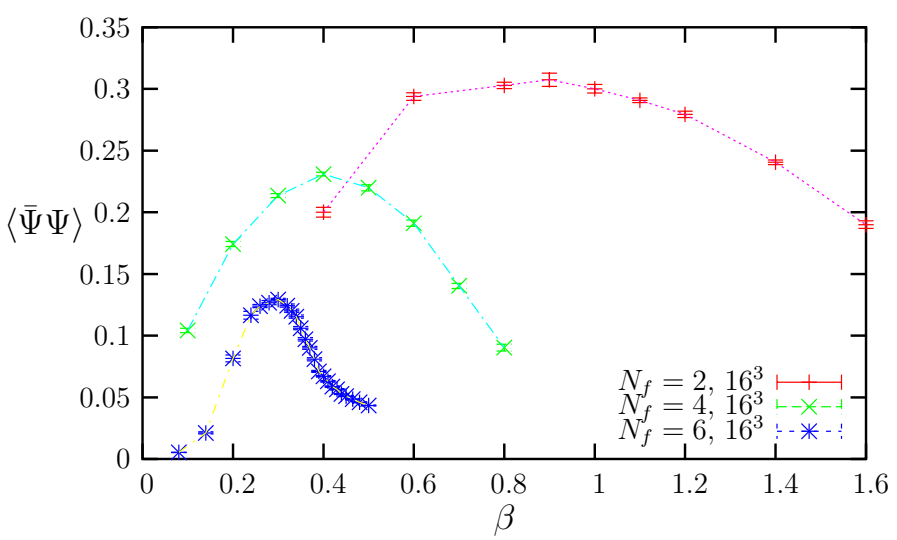

Figure 3: Chiral condensate vs. $\beta$ for $N_{f}=2,4,6$ on $16^{3}$ lattices with $m=0.01$.

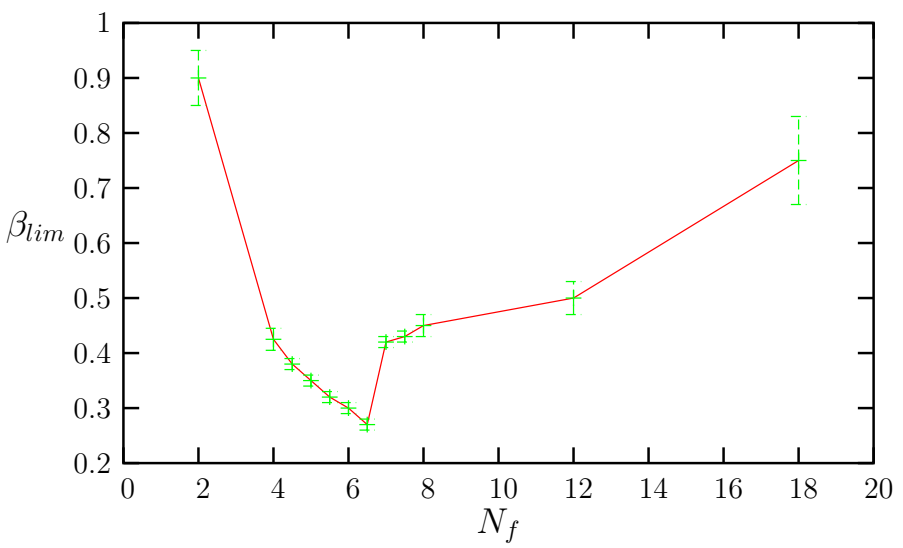

Figure 4: $\beta_{\lim }$ vs. $N_{f}$.

$g_{R}^{2} \in[0, \infty)$, ie. for $g^{2} \in\left[0, g_{\lim }^{2}\right)$; to leading order in $1 / N 1 / g_{\lim }^{2}=2 / 3$ for $m=0$. In summary, the strong coupling limit $1 / g^{2} \rightarrow 0$ of the continuum model is recovered in the lattice model at $1 / g^{2} \rightarrow g_{\text {lim }}^{2}>0$. As we already discussed in the Introduction, chiral symmetry breaking is absent in large- $\mathrm{N}$ calculations. Therefore, it may well be that the value of the second diagram in Fig. 1 is considerably altered in a chirally broken vacuum. Later in this section we will use this strong coupling lattice discretization effect as a criterion to decide whether chiral symmetry is broken or not at infinite coupling for different values of $N_{f}$. The data presented in Fig. 2 1 show clearly that the value of $\beta_{\lim }$ (which corresponds to the infinite coupling limit in the continuum), where the condensate has its maximum value does not depend on the lattice size, because as mentioned in an earlier paragraph this is an $O\left(a^{-1}\right)$ discretization effect. In addition, the same figure shows that within our statistical errors the position of the peak does not depend on the lattice size or the bare mass. Figure 3 shows that as expected the value of the chiral condensate (for $m=0.01$ ) decreases as $N_{f}$ is increased, because the fermion-antifermion screening has a stronger contribution for larger $N_{f}$. Furthermore, the value of $\beta_{\text {lim }}$ shifts to the left as the number of fermion flavors increases from $N_{f}=2$ to $N_{f}=6$. Figure $₫$ shows that this trend is not monotonic; near $N_{f} \approx 6.5$, the curve of 


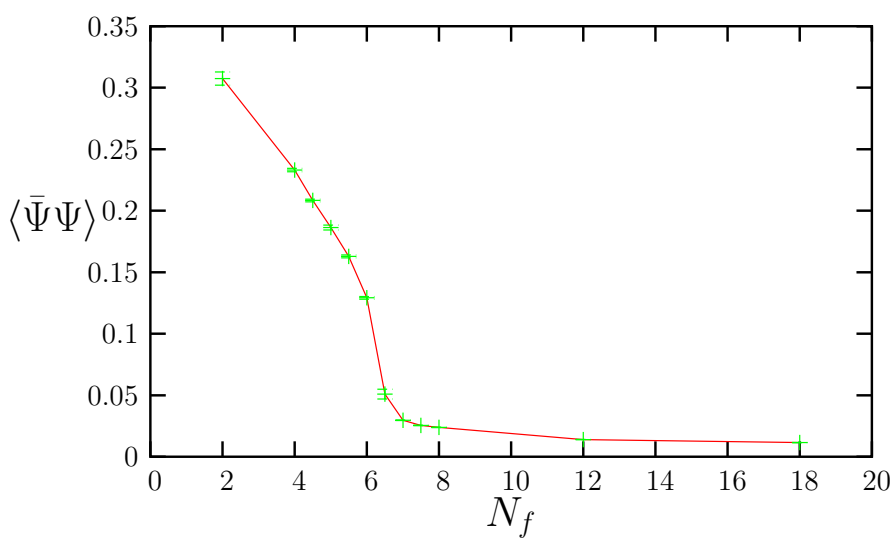

Figure 5: Chiral condensate at $\beta_{\text {lim }}$ for different values of $N_{f}$.

$\beta_{\text {lim }}\left(N_{f}\right)$ reaches a minimum, implying a significant change in the strong coupling behavior of the model. The minimum in $\beta_{\lim }\left(N_{f}\right)$ is a significant change in the phase diagram of the $3 \mathrm{~d}$ Thirring model, implying that $N_{f c} \approx 6.5$. This conclusion is enhanced by the results shown in Fig. 5, where we plot the chiral condensate at $\beta_{\lim }\left(N_{f}\right)$ versus $N_{f}$. The relatively rapid change in the value of the condensate around $N_{f}=6.5$ may imply that a phase transition separates a chirally broken from a chirally symmetric phase with $N_{f c} \approx 6.5$. We are currently extending our simulations in order to understand better the properies of the chiral symmetry restoration transition.

\section{Analysis of $N_{f}=6$ data}

During the last decade, Monte Carlo simulations provided evidence that for $N_{f} \leq 5$ and for sufficiently strong coupling the $3 \mathrm{~d}$ Thirring model is in the chirally broken phase [8,, , 10, 11]. In this section we present results from new simulations with $N_{f}=6$ on lattice sizes varying from $12^{3}$ to $32^{3}$. As expected the phase transition for $N_{f}=6$ is steeper than the transitions with smaller $N_{f}$. However, a detailed finite size scaling analysis of the Binder's Cumulant (not shown here) showed that the data at $m=0.01$ are consistent with a crossover instead of a first order transition. In order to understand the properties of the model near the transition we fitted the data for the chiral condensate extracted from simulations on the largest $32^{3}$ lattices to the Equation of State (EoS):

$$
m=A\left(\beta-\beta_{c}\right)\langle\bar{\chi} \chi\rangle^{p}+B\langle\bar{\chi} \chi\rangle^{\delta}
$$

where $p=\delta-1 / \beta_{m}$. The assumption in eq. (4.1) that finite size effects are negligible is justified by the observation that for the smallest mass used, $m=0.01$, the difference between the values of $\langle\bar{\Psi} \Psi\rangle$ extracted from simulations on $24^{3}$ and $32^{3}$ lattices is small. The fit was performed for $\beta=$ $0.34, \ldots, 0.44$ (see fig. 6), i.e. close enough to the transition but still away from $\beta_{\text {lim }} \approx 0.30$ where strong coupling lattice discretization effects are severe. The results are: $A=1.38(13), B=58(25)$, $\beta_{c}=0.3082(36), \delta=4.65(27)$, and $\beta_{m}=0.28(2)$ with an acceptable fit quality $\chi^{2} /$ dof $=1.6$. For $N_{f}=6$ the value $\beta_{c}$ is close to $\beta_{\text {lim }}$, implying that $N_{f}=6$ is close to $N_{f c}$, which is in accordance with the results presented in section 4 . We also performed fits which included data from smaller 


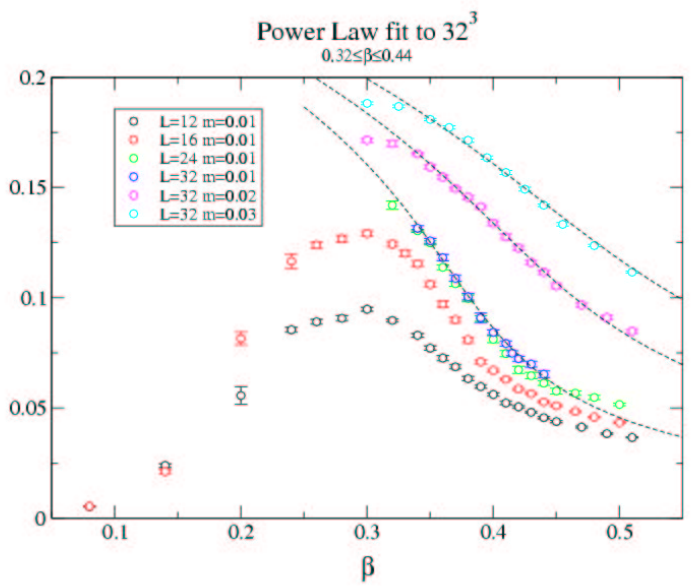

Figure 6: Chiral condensate vs. $\beta$ for $N_{f}=6$. The dashed lines represent fits of the $32^{3}, 0.32 \leq \beta \leq 0.44$ data to eq. 4.1 .

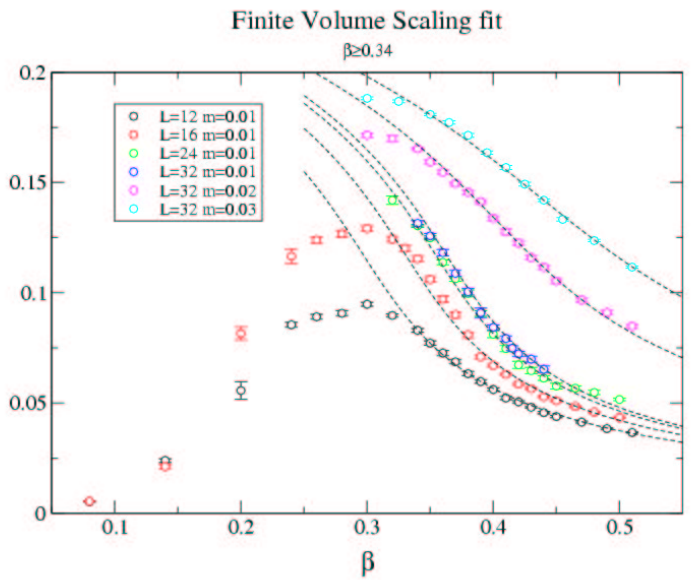

Figure 7: Chiral condensate vs. $\beta$ for $N_{f}=6$. The dashed lines represent fits of data from all lattice sizes for $\beta \geq 0.34$ to eq. 4.2 .

volumes to a finite size scaling form of the EoS:

$$
m_{0}=A\left(\left(\beta-\beta_{c}\right)+C L^{-\frac{1}{v}}\right)\langle\bar{\chi} \chi\rangle^{p}+B\langle\bar{\chi} \chi\rangle^{\delta}
$$

The results extracted from fits to eq. (4.2) (see fig. $\nabla$ ) are of lower quality $\left(\chi^{2} / d o f=5.8\right.$ ) because on smaller volumes the peaks near $\beta_{\text {lim }}$ are broader, implying that strong coupling lattice discretization effects are more severe. In this case we got $A=1.91(4), B=231(53), \beta=0.3185(10)$, $\delta=5.49(13)$, and $\beta_{m}=0.19(2)$. 


\section{Conclusions}

Our main results is that the $N_{f}=63 \mathrm{~d}$ Thirring model like its smaller $N_{f}$ counterparts appears to undergo a second order chiral symmetry breaking phase transition at strong coupling. Moreover, the $N_{f}=6$ critical exponents are distinct from those of $N_{f}=2$ and $N_{f}=4$ i.e. the different models have interacting continuum limits which are qualitatively, but not quantitatively similar. Further, a detailed analysis of the strong coupling behavior of the model for $N_{f}=2, \ldots, 18$ revealed that the critical number of fermion flavors where chiral symmetry is restored is $N_{f c} \approx 6.5$ which is distinctly larger than the $N_{f c}=4.32$ predicted by certain SDE approaches [1] and it contradicts the conclusion drawn from other SDE approaches [2] which predict chiral symmetry breaking for all values of $N_{f}$. Our results may have broader interesting implications on the applicability of SDEs in non-perturbative field theories. There are predictions [3, 4, 5] that in the strong coupling limit the vector meson becomes massless suggesting that the IR limit of $\mathrm{QED}_{3}$ coincides with the UV limit of the $3 \mathrm{~d}$ Thirring model. The problem of detecting dynamical symmetry breaking in lattice $\mathrm{QED}_{3}$ is much harder than in $3 \mathrm{~d}$ Thirring, because in $\mathrm{QED}_{3}$ the dynamical fermion mass is much smaller than the momentum cut-off [12].

\section{Acknowledgements}

The simulations were performed on a cluster of 64-bit AMD Opterons 250 at the Frederick Institute of Technology, Cyprus.

\section{References}

[1] T. Itoh, Y. Kim, M. Sugiura and K. Yamawaki, Prog. Theor. Phys. 93 (1995) 417 [hep-th/9411201].

[2] D.K. Hong and S.H. Park, Phys. Rev. D49 (1994) 5507 [hep-th/9307186].

[3] G. Parisi, Nucl. Phys. B100 (1975) 368; S. Hikami and T. Muta, Prog. Theor. Phys. 57 (1977) 785; Z. Yang, Texas preprint UTTG-40-90 (1990).

[4] M. Gomes, R.S. Mendes, R.F. Ribeiro and A.J. da Silva, Phys. Rev. D43 (1991) 3516.

[5] S.J. Hands, Phys. Rev. D51 (1995) 5816 [hep-th/9411016].

[6] T. Appelquist, M. Bowick, D. Karabali and L.C.R. Wijewardhana, Phys. Rev. D33 (1986) 3704, 3774; M.R. Pennington and D. Walsh, Phys. Lett. B253 (1991) 246; P. Maris, Phys. Rev. D54 (1996) 4049 [hep-ph/9406214].

[7] V.A. Miranskii, Nuovo Cimento 90A (1985) 149.

[8] L. Del Debbio and S. Hands, Phys. Lett B373 (1996) 171 [hep-lat/9512013].

[9] L. Del Debbio, S. Hands, and J.C. Mehegan, Nucl. Phys. B502 (1997) 269 [hep-1at/9701016].

[10] L. Del Debbio and S. Hands, Nucl. Phys. B552 (1999) 339 [hep-lat/9902014].

[11] S. Hands and B. Lucini, Phys. Lett. B461 (1999) 263 [hep-lat/9906008].

[12] S. Hands, J. Kogut, C. Strouthos, Nucl. Phys. B645 (2002) 321 [hep-lat/0208030]; S. Hands, J. Kogut, C. Strouthos, Phys. Rev. B70 (2004) 104501 [hep-lat / 0404013 ]. 